\title{
${ }_{\text {Surface Drift Currents Induced by Waves and Wind in a Large Tank }}$
}

\author{
VladisLAV POLNIKOV \\ A.M. Obukhov Institute of Atmospheric Physics, Russian Academy of Sciences, Moscow, Russia
}

FANGLI QIAO

First Institute of Oceanography, Ministry of Natural Resources, and Qingdao National Laboratory for Marine Science and Technology, Qingdao, China

\author{
HONGYU MA \\ First Institute of Oceanography, Ministry of Natural Resources, and Key Laboratory of Marine Science and Numerical Modeling, \\ Ministry of Natural Resources, Qingdao, China
}

(Manuscript received 30 January 2020, in final form 5 August 2020)

\begin{abstract}
The empirical features of surface drift currents induced by both mechanical and wind waves are presented. The measurements were made by using surface floats in a large tank with dimensions of $32.5 \times 1 \times 2 \mathrm{~m}^{3}$. Three cases were studied: (i) regular (narrowband) mechanical waves, (ii) irregular (wideband) mechanical waves, and (iii) wind waves. The measured surface drift currents induced by mechanical waves $U_{d}$ are compared with the Stokes drift at the surface $U_{\mathrm{St}}$ estimated by a well-known formula with an integral over the wave spectrum. In this case, the ratio $U_{d} / U_{\mathrm{St}}$ varies in the range of $0.5-0.93$ and slightly increases with decreasing wave steepness. No visible dependence on the breaking intensity is observed. In the case of wind waves, the wind-induced part of the surface drift $U_{d w}$ is compared with the friction velocity $u_{*}$. In our measurements, the ratio $U_{d w} / u_{*}$ varies systematically in the range of $0.65-1.2$. Considering the percentage of wave breaking $\mathrm{Br}$, the wave age $A$, and the wave steepness $\sigma$, the parameterization of $U_{d w}$ was obtained in the form $U_{d w}=(\mathrm{Br}+\sigma A) u_{*}$, which corresponds to the observations with a mean error of $10 \%$. For the first time, this ratio provides the dependence of windinduced drift on the surface wave parameters. The obtained results and problems related to measuring surface drift currents are discussed.
\end{abstract}

KEYWORDS: Air-sea interaction; Small scale processes; Surface fluxes

\section{Introduction}

\section{a. Initial information}

Surface drift currents play a significant role in marine shipping, the spread of surface waste, and marine industry safety. Thus, studying and understanding this phenomenon, which was comprehensively described in the recent review by Bremer and Breivik (2017), is of physical and practical importance. From the physical perspective, the drift currents are interesting due to their observability, availability for measurements, and possibility of theoretical description (e.g., Stokes 1847; LonguetHiggins 1953; Craik 1982; Bremer and Breivik 2017, and references therein). For this reason, a large number of surface drift current measurements have been obtained over long periods, started by Shemdin (1972), Wu (1975, 1983), Churchill and Csanady (1983), and Bye (1988), and continued at present (e.g., Longo 2012; Longo et al. 2012; Zavadsky and Shemer 2017). The earlier work was well described by Tsahalis (1979), and an extended list of recent work was reported by Bremer and Breivik (2017). In addition, extensive theoretical work has

¿ Denotes content that is immediately available upon publication as open access.

Corresponding author: Vladislav Polnikov, polnikov@mail.ru addressed numerous aspects of the dynamics of currents induced by waves in water, namely, the generation of current vorticity due to viscosity, particle trajectories in water, turbulence induced by waves, the oscillation of boundary layers, the occurrence of return currents in a tank, the origin of Langmuir circulations, and so on. Most of these topics have been well described in certain papers (e.g., Longuet-Higgins 1953; Craik 1982; Teixeira and Belcher 2002; Babanin and Haus 2009; Teixeira 2018), surveys (e.g., Sullivan and McWilliams 2010; Bremer and Breivik 2017), and monographs (e.g., Phillips 1977; Janssen 2004; Bühler 2014). However, in this paper, we consider measurements of time-averaged surface currents induced by waves in water. The focus of the study is on the experimental aspects of the surface current problem rather than particle trajectories near the surface, turbulence generation by waves, or the depth profile of induced currents.

Despite the evident simplicity of surface drift current measurements, accurate measurements of a wavy water surface can have many sources of error. Field measurements (e.g., Churchill and Csanady 1983; Babanin 1988; Malinovsky et al. 2007; Kudryavtsev et al. 2008) are influenced by the nonstationarity of the wind field, uncontrolled background currents $U_{b}$, the stratification of air and water in situ, numerous technical difficulties in field measurements, and other factors. In laboratory (tank) measurements 
(Toba 1973; Wu 1975; Tsahalis 1979; Longo 2012; Longo et al. 2012; Paprota et al. 2016; Zavadsky and Shemer 2017), the sources of errors include the influence of the upper and lateral boundaries of the tank, presence of return currents, short wind and wave fetches, and difficulty of placing equipment in a tank. Some specific problems related to surface current measurements will be discussed in this paper.

All these limitations influence the wind and current profiles and establish dependencies of the surface drift on the wind and wave parameters of the air-water interface. Besides, small tank dimensions limit the range of wave state parameters, thereby decreasing the ability to identify the abovementioned dependencies. However, tank measurements have the evident advantage that all parameters in the air-water system can be completely controlled during an experiment. Therefore, the most exact and reliable results for surface drift currents have been obtained in laboratory measurements (Wu 1975, 1983; Longo 2012; Longo et al. 2012; Zavadsky and Shemer 2017). Nevertheless, many features of the drift current phenomenon are still not completely understood. In this paper, some of these issues will be addressed based on our own tank measurements.

\section{b. Specification of the tasks}

For completeness, consider two principally different cases: (i) mechanical waves and (ii) wind waves in water.

Regarding mechanical waves, one may expect that the surface drift current $U_{d}$ should be of the same order as the Stokes mass-transport current $U_{\mathrm{St}}$ if the ideal fluid assumptions are applicable (Stokes 1847). In the potential approximation and the case of a monochromatic nonlinear gravity wave with amplitude $a$, frequency $\omega$, and wavenumber $k=\omega^{2} / g(g$ is the gravity acceleration), the theory predicts the ratio (Stokes 1847; Phillips 1977)

$$
U_{\mathrm{St}}=(\omega / k)(k a)^{2}=(\omega a)(k a)
$$

where the first equality corresponds to the scaling of $U_{\mathrm{St}}$ by the phase velocity, $\omega / k$, and the second equality corresponds to the scaling of $U_{\mathrm{St}}$ by the orbital velocity, $\omega a$. The factor $k a$ is the steepness of the wave (typically $\sim 0.1$ ), which determines the scale of the Stokes drift with respect to the mentioned velocities. For the wave spectrum $S(\omega)$, Eq. (1) for $U_{\text {St }}$ can be generalized. In the case of quasi-monochromatic (narrowband) waves, Eq. (1) can be replaced by the following formula:

$$
U_{\mathrm{St}}=\omega_{p} k_{p} a^{2},
$$

where $\omega_{p}$ and $k_{p}$ are the spectral peak frequency and the peak wavenumber, respectively. In the case of mechanical waves with wideband spectra, such as Pierson-Moscowitz (PM) or Joint North Sea Wave Observation Project (JONSWAP; JW) spectra (Komen et al. 1994), the Stokes drift can be estimated by the following formula (Churchill and Csanady 1983):

$$
U_{\mathrm{St}}(z)=(2 / g) \int_{\omega_{\min }}^{\omega_{\max }} \omega^{3} S(\omega) \exp \left(2 \omega^{2} z / g\right) d \omega
$$

Here, $\left[\omega_{\min }, \omega_{\max }\right]$ is the available frequency band of the wave spectrum $S(\omega)$, and $z$ is the vertical coordinate directed positively upward. Equation (3) allows the Stokes drift for a given spectrum $S(\omega)$ to be estimated at both the surface and at any depth $z$; besides, it shows that the Stokes drift decays exponentially with depth $z$. Note that ratios (2) and (3) are also used for the estimation of $U_{\mathrm{St}}(0)$ in the presence of wind waves ( $\mathrm{Wu}$ 1983; Toba 1973; Clarke and Gorder 2018).

Unfortunately, direct measurements of real surface drift currents $U_{d}$, induced by mechanical waves, are rather limited (according to Bremer and Breivik 2017). For example, Craik (1982) described the depth profile formation of currents induced by different kinds of waves in viscous and contaminated water. Herterich and Hasselmann (1982) provided results for the diffusion of tracers by surface waves. Monismith et al. (2007) presented results for return current formation in a water body of finite depth. Paprota et al. (2016) and Breivik et al. (2014) measured Stokes drift profiles. In all these works, the authors noted a discrepancy between the measured currents $U_{\mathrm{St}}(z)$ and the theoretical results (e.g., Longuet-Higgins 1953; Craik 1982; Constantin 2006). However, none of the researchers did quantify the measured surface drift current $U_{d}$ in comparison with the theoretical value $U_{\mathrm{St}}(0)$. This relation needs further specification, and this issue will be addressed in this paper based on tank measurements.

In the case of wind waves, the total surface drift (in the absence of background currents) includes two terms (the case of one-dimensional tank measurements is assumed):

$$
U_{d}=U_{\mathrm{St}}(0)+U_{d w},
$$

where $U_{d w}$ is the wind-induced part of the surface drift current $U_{d}$. To estimate $U_{d w}$, the Stokes drift needs to be excluded from $U_{d}$ in Eq. (4); to this aim, the values of $U_{\mathrm{St}}(0)$ are often estimated using Eq. (2). Based on such estimates, previous studies (Wu 1975, 1983; Churchill and Csanady 1983; Longo 2012; Longo et al. 2012; Zavadsky and Shemer 2017) showed that the Stokes drift at the surface $U_{\mathrm{St}}(0)$ has a typical empirical value of approximately $10 \%-15 \%$ of the wind-induced drift $U_{d w}$. This indicates that $U_{\mathrm{St}}$ should be taken into account in measurements and practical tasks, especially in the presence of intensive long waves that have high phase velocities (e.g., swell). Moreover, in the field measurements by Clarke and Gorder (2018), the Stokes drift was considered as the total surface drift current, with no attention given to the windinduced part $U_{d w}$.

In practice, the wind-induced drift currents $U_{d w}$ have values on the order of the friction velocity in air $u_{*_{a}}$. With no swell, such currents can be described by the simple ratio (Wu 1975; Longo et al. 2012; Zavadsky and Shemer 2017)

$$
U_{d w}=c_{d} u_{* a},
$$

where coefficient $c_{d}$ varies in the range from 0.3 in the laboratory measurements (Zavadsky and Shemer 2017) to 0.53 (Wu 1975), and from 0.2 (Babanin 1988) to 1.5 (Tsahalis 1979) in the field measurements. In all cases, no authors considered the dependence of $U_{d w}$ on specific wave parameters. In the present paper, we address this relation in detail. 


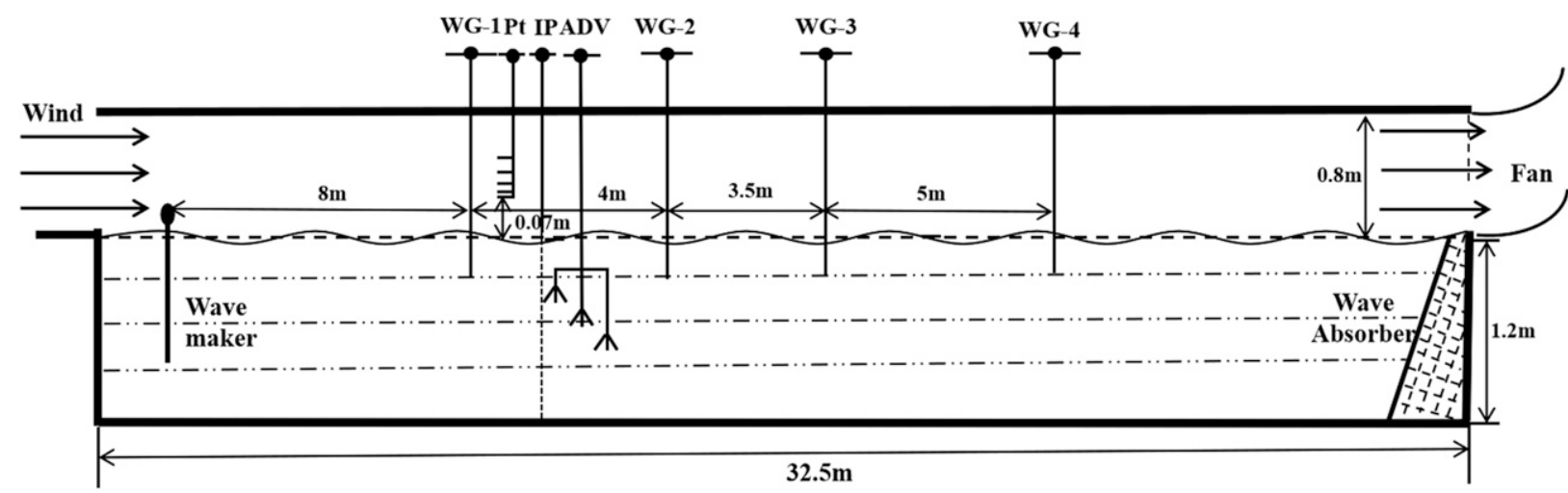

FIG. 1. A sketch of the tank. WGs are the wave gauges, PT are the Pitot tubes, ADV is the set of the acoustic Doppler velocimeters, and IP is the set for injecting in water float particles or a color dye. The width of the tank is $1 \mathrm{~m}$.

Until recently, it was unclear why the surface drift current $U_{d w}$ has values on the order of the friction velocity in air $u_{* a}$ rather than the friction velocity in water, $u *_{w} \approx 0.03 u_{*_{a}}$, as should follow from the condition of momentum-flux continuity at the interface. Second, it was unknown why the empirical ratio $U_{d} / u_{*_{a}}$ does not manifest any dependence on wave parameters. Despite of numerous theoretical work in this field (e.g., Huang 1979; Kitaigorodskii et al. 1983; Lumley and Terray 1983; Anis and Moum 1995; Mellor 2001; Rascle and Ardhuin 2009; Chalikov and Rainchik 2011; Babanin and Chalikov 2012; Benilov 2012; Teixeira 2018, and references therein), the answer to these questions was found only in the recent paper by Polnikov (2019).

In Polnikov (2019), a simple semi-phenomenological model was constructed based on the theoretical concept of the threelayer structure of the air-water interface (Polnikov 2011). Processing the numerical simulation data obtained by Chalikov and Rainchik (2011) and Polnikov (2011) showed that the profile of the total drift current, $U_{d}(z)$, corresponding to Eq. (4), is linearly related to depth $z$ in the so-called "wave zone," where the air and the water alternatively exist. This finding was empirically confirmed by the measurements of Longo (2012) and Longo et al. (2012) (see details in Polnikov 2019). This means that the wave zone plays the role of a friction layer between the air and the water layers.

Polnikov (2019) proposed that in the wave zone the following ratio is valid

$$
\tau_{t w} / \rho_{w}=K_{t} \frac{\partial U_{d w}(z)}{\partial z}
$$

where $\tau_{t w} / \rho_{w} \simeq 0.8 u_{*_{a}}^{2}$ is the vertical wind-induced momentum flux in the water, normalized by the water density $\rho_{w}$ and related to drift current production; $U_{d w}(z)$ is the windinduced drift; $K_{t}=f_{t}(.) U_{d} h$ is the effective eddy viscosity in the water part of the wave zone, where $h$ is the wave height and $f_{t}($.$) is a dimensionless fitting function that depends on$ surface wave parameters [the symbol (.) indicates the possible dependence of function $f$ on the parameters of the interface]. As the values $\tau_{t w}$ and $\partial U_{d w}(z) / \partial z$ are constant in the wave zone, Eq. (6) yields

$$
U_{d w}=\left[C_{p} / f_{t}(.)\right]^{1 / 2} u_{*_{a}},
$$

where $C_{p}$ is a constant of the order of air-to-water density ratio $\rho_{a} / \rho_{w}$. Thus, the empirical ratio (5) describing the relation between $U_{d w}$ and $u_{*_{a}}$ is theoretically justified. If the values of $f_{t}($.$) vary within the range of 0.01-0.03$, ratio (7) quantitatively coincides with ratio (5) [note that in the above consideration, the smallness of $U_{\mathrm{St}}(0)$ with respect to $U_{d w}$ is taken into account].

One may expect that the wind-induced drift velocity $U_{d w}$ is dependent on the following dimensionless parameters of the wave state: the steepness $\sigma=a k_{p}$, the wave age $A=g /\left(\omega_{p} u_{*}\right)$, and the breaking intensity Br. It was suggested in Polnikov (2019) that this dependence could be "hidden" in the fitting function $f_{t}($.$) . However, the function f_{t}($.$) cannot be theoretically derived$ because it has a turbulent (statistical) nature. Therefore, the proposed assumption must be empirically verified.

Based on our own direct drift current measurements obtained with surface floats in a tank, the above dependencies were successfully revealed. In parallel, we quantified the ratio between the measured surface drift currents $U_{d}$, induced by mechanical waves in water, and the expected Stokes drift $U_{\mathrm{St}}(0)$, provided by Eq. (3). Obtaining a solution for the first task is the main objective of our experiment, while the second task is an auxiliary consideration following from the limitations of the studies listed above. On this way, we encountered some methodological difficulties related to measuring the surface drift currents with floats, and these issues are worthy of discussion.

The layout of the paper is as follows. Section 2 describes the equipment and measurement methods used. The results and analysis for mechanical waves and wind waves are presented in sections 3 and 4, respectively. Section 5 contains a general discussion, and the conclusions are given in section 6 .

\section{Equipment and measurement description}

In our experiment, we used the wind-wave tank of the First Institute of Oceanography of the Ministry of Natural Resources (FIO) located in Qingdao, China. A sketch of the tank is shown in Fig. 1. 
For the wave measurements, capacity wave gauges (WGs) were used. Pitot tubes (PTs) and three acoustic Doppler velocimeters (ADV) were used to measure the wind profile and current fluctuations, respectively. The dimensions of the tank, water depth, and air space, as well as the equipment locations, are given in Fig. 1. The locations of WG1-WG4 are denoted below as points $\mathrm{P} 1-\mathrm{P} 4$.

The wind is provided by the fan located at the outlet of the tank. Closed air circulation is designed (not shown). The PT set has five channels located at horizons of 7,10,14,25, and $40 \mathrm{~cm}$. For winds greater than $10 \mathrm{~m} \mathrm{~s}^{-1}$, all the PTs were located $1.2 \mathrm{~cm}$ above these levels.

Two kinds of mechanical waves were studied: (i) regular quasi-monochromatic waves and (ii) stochastic waves with a wide spectral band similar to those of PM or JW wave spectra (Komen et al. 1994). These waves were generated by a wavemaker with three initial dominant frequencies $f_{0}$ of $1.5,1.0$, and $0.7 \mathrm{~Hz}$ and 3-5 amplitudes at each initial frequency, ranging from $H_{S}=1 \mathrm{~cm}$ to $H_{S}=20 \mathrm{~cm}$ (here, $H_{S}$ is the programmed significant wave height for generated waves). All these parameters were established in the wavemaker computer.

As another type of wave, wind waves were generated by the fan. The fan wind $W$ is approximately equal to the mean wind speed at the middle of the air part of the tank (i.e., at $z=$ $40 \mathrm{~cm}$ ). Five values of wind $W$ were applied: 4, 6, 8, 10, and $12 \mathrm{~m} \mathrm{~s}^{-1}$.

The wave records were $10 \mathrm{~min}$ long with a sampling frequency of $50 \mathrm{~Hz}$, and each run had different wave or wind parameters. The same duration was used for the wind measurements with PT, for which the record averaging over 1-min intervals was performed. The measurements of the surface currents were obtained during these $10 \mathrm{~min}$ for each run. The 10-min measurements of the current fluctuations in the water body (at three horizons: $-10,-20$, and $-30 \mathrm{~cm}$ ) were made by an ADV with a sampling of $100 \mathrm{~Hz}$. The description of these fluctuations is beyond the scope of this paper and will be presented in a separate work.

All the measurements were made 5 min after switching on the wavemaker or the fan to allow for system stabilization. In total, 42 runs were executed.

The surface drift current was measured based on the visual timing of the surface floats passing through the fixed length $L$. The surface floats were spherical plastic balls with bright colors (red, orange, and yellow, to distinguish them on the water surface) and two different diameters of 3 and $5 \mathrm{~mm}$. The specific gravity of the floats was approximately $500 \mathrm{~kg} \mathrm{~m}^{-3}$. The fixed length $L$ varied from 1 to $5 \mathrm{~m}$ based on the expected drift velocity. For each run, the floats' timing was recorded 3-7 times until getting a minimal scattering of the results. An acceptable scattering range is approximately $5 \%-10 \%$ of the mean timing, which defines the empirical accuracy of the driftcurrent measurements.

For mechanical waves, the drift measurements were made at two drift-starting points: $\mathrm{P} 1=9 \mathrm{~m}$ and $\mathrm{P} 2=12 \mathrm{~m}$. It was assumed that the drift current features for mechanical waves depend weakly on location. The most intensive measurements were made at point $\mathrm{P} 2$; the measurements at point $\mathrm{P} 1$ were mainly made to check the homogeneity of the current field.
For wind waves, intensive measurements were made at three points: P1, P2, and P4 $=22 \mathrm{~m}$. Small shifts in the locations of $\mathrm{P} 1, \mathrm{P} 2$, and $\mathrm{P} 4$ from the actual locations of WGs occurred due to technical reasons.

The floats were injected to the tank space with special equipment, IP, which included a $3-\mathrm{mm}$ aluminum tube that was vertically fixed at the roof of the tank and continued to the level of $10 \mathrm{~cm}$ above the still water surface. Inside this tube, there was a $1 \mathrm{~mm}$ (capillary) flexible tube that was used to inject dye into the water at a fixed depth to study the wave-induced turbulent diffusion (this topic is beyond the scope of this paper). For each set of drift measurements, 2-3 plastic floats were simultaneously added to the tank at a point located $5-10 \mathrm{~cm}$ in front of the drift-starting point for each run. Several technical problems related to using spherical floats in this task will be discussed in section 5 . The processing of the drift current data was performed without any software, as no records were collected with the equipment used.

The wave and wind data were processed with the MATLAB software. For the wave spectra evaluation, the autoregression (AR) and Welch methods were used. Because the data series had 30000 points (more than 1000 dominant periods), the $95 \%$ confidence intervals were very small (approximately 1500 degrees of freedom). In the bilogarithmic coordinates, they were $[+10 \%,-12 \%]$ and $[+15 \%,-20 \%]$ for the AR method and the Welch method, respectively. The frequency spectra $S(f)$, shown in this paper, were estimated with the AR method and are presented based on the circular frequency $f$ given in hertz (instead of the theoretical giving spectrum in the radian frequency, $\omega=2 \pi f$ ) (shown in Fig. 2).

The intensity of wave breaking (in percent) was visually fixed based on the relative numbering of the broken wave crests with respect to the total number of dominant crests. This numbering process was executed by two persons in parallel watching the dominant crests moving between points $\mathrm{P} 2=12 \mathrm{~m}$ and $\mathrm{P} 4=22 \mathrm{~m}$ during one minute of observation just before the main measurements were made. The intensity of the microbreaking of small-size crests was not fixed.

\section{Mechanical waves: Results and analysis}

Regular (narrowband) and irregular (wideband) mechanical waves were considered separately. The results for the first kind of wave are described in detail, and these details are not repeated for the second kind of wave. The analysis of all the results is combined.

\section{a. Results for regular waves}

The results are summarized in Table 1 . First, we should note a reasonable (up to $30 \%$ ) difference between the estimations of the surface Stokes drift $U_{\mathrm{St}}$ found with Eqs. (2) and (3) (hereafter, the notation of the surface level, $z=0$, is omitted for simplicity). An example of the relation between these estimates of $U_{\mathrm{St}}$ for regular waves is shown in Fig. 3a, and an example of the spectrum for narrowband waves, used to get the above estimations, is shown in Fig. 2a. Importance of using Eq. (3) is evident. Notably, in the integral of Eq. (3), we restricted the upper boundary at $f_{\max }=15 \mathrm{~Hz}$ due to the accuracy restrictions of the WGs with a 1-mm wave height. The 

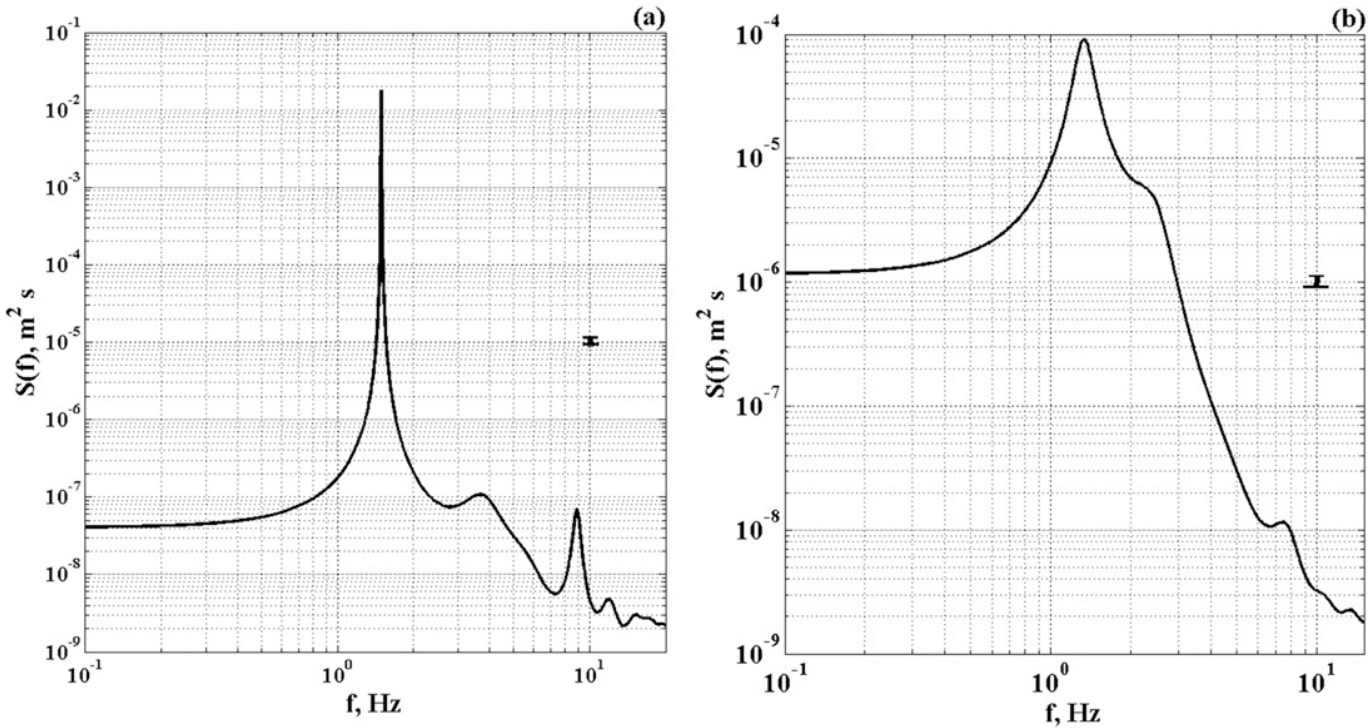

FIG. 2. Spectra at point P2 for waves with $H_{s}=3 \mathrm{~cm}, f_{p}=1.5 \mathrm{~Hz}$ : (a) regular waves and (b) irregular waves. The $95 \%$ confidence intervals, shown by small bars, are on the order of $[+10 \%,-12 \%]$.

difference between the mentioned estimates suggests that Eq. (3) is the more accurate equation. Hereafter, only such estimations of $U_{\mathrm{St}}$ are presented.

The second peculiarity of the measurements is that the ratio $U_{d} / U_{\mathrm{St}}$ varies in the range of $0.5-0.8$ (the second-to-last column in Table 1), reflecting a permanent underestimation of the expected surface Stokes drift. Specifically, there is no evident dependence of this ratio on the intensity of wave breaking (presented in the rightmost column in Table 1). Following the review by Bremer and Breivik (2017), the estimates of $U_{d} / U_{\mathrm{St}}$ for the tank measurements are pioneering, despite numerous empirical reports of the abovementioned discrepancies between measurements and theoretical values. A more detailed analysis will be made in section $3 \mathrm{c}$.

\section{b. Results for irregular waves}

These results are summarized in Table 2. In this case, the peculiarities are similar to those in the previous case. The role of spectral shape is well shown in Figs. 2a,b and 3a,b. From these figures, one can see that for a wideband spectrum, the estimates of $U_{\text {st }}$ based on Eqs. (2) and (3) can be more than 2 times different.

The additional difference in the surface drift for irregular waves is a slightly greater ratio $U_{d} / U_{\mathrm{St}}$ varying from 0.6 to 0.9 . In this case, the integral in Eq. (3) should be greater due to the

TABLE 1. Parameters of waves and surface currents for regular waves. Note that the two values for the Stokes drift, separated by a slash, correspond to the estimations with Eqs. (2) and (3), respectively; "n-dim" indicates nondimensional.

\begin{tabular}{|c|c|c|c|c|c|c|c|c|c|}
\hline $\begin{array}{c}\text { Paddle } \\
H_{S}(\mathrm{~cm})\end{array}$ & $\begin{array}{c}\text { Wave } \\
\text { amplitude } \\
a(\mathrm{~cm})\end{array}$ & $\begin{array}{c}\text { Peak } \\
\text { frequency } \\
f_{p}(\mathrm{~Hz})\end{array}$ & $\begin{array}{c}\text { Peak } \\
\text { frequency } \\
\omega_{p}\left(\mathrm{~s}^{-1}\right) \\
\end{array}$ & $\begin{array}{c}\text { Peak } \\
\text { wavenumber } \\
k_{p}\left(\mathrm{~m}^{-1}\right)\end{array}$ & $\begin{array}{l}\text { Steepness } \\
\sigma(\text { n-dim })\end{array}$ & $\begin{array}{l}\text { Stokes drift } \\
U_{\mathrm{St}}\left(\mathrm{cm} \mathrm{s}^{-1}\right)\end{array}$ & $\begin{array}{l}\text { Mean drift } \\
U_{d}\left(\mathrm{~cm} \mathrm{~s}^{-1}\right)\end{array}$ & $\begin{array}{c}U_{d} / U_{\mathrm{St}} \\
(\mathrm{n}-\mathrm{dim})\end{array}$ & $\begin{array}{c}\text { Intensity of } \\
\text { breaking (\%) }\end{array}$ \\
\hline \multicolumn{10}{|c|}{ Paddle frequency $f_{p}=1.5 \mathrm{~Hz}$} \\
\hline 3 & 1.36 & 1.49 & 9.36 & 8.9 & 0.12 & $1.5 / 1.7$ & 1.35 & 0.79 & No breaking \\
\hline 5 & 2.13 & 1.49 & 9.36 & 8.9 & 0.19 & $3.8 / 4.1$ & 3.1 & 0.76 & $<10 \%$ \\
\hline 7 & 2.76 & 1.485 & 9.33 & 8.9 & 0.25 & $6.4 / 7.8$ & 5.6 & 0.72 & $30 \%$ \\
\hline 10 & 3.20 & 1.48 & 9.29 & 8.8 & 0.28 & $8.3 / 11.7$ & 9.1 & 0.78 & $50 \%$ \\
\hline 15 & \multicolumn{9}{|c|}{ Data are not reliable due to bad wave formation } \\
\hline \multicolumn{10}{|c|}{ Paddle frequency $f_{p}=1.0 \mathrm{~Hz}$} \\
\hline 3 & 1.03 & 1.0 & 6.28 & 4.0 & 0.041 & $0.27 / 0.36$ & 0.26 & 0.72 & No breaking \\
\hline 5 & 1.74 & 1.0 & 6.28 & 4.0 & 0.070 & $0.77 / 0.98$ & 0.71 & 0.72 & - \\
\hline 7 & 2.45 & 1.0 & 6.28 & 4.0 & 0.10 & $1.54 / 1.90$ & 1.43 & 0.75 & - \\
\hline 10 & 3.48 & 1.0 & 6.28 & 4.0 & 0.14 & $3.1 / 3.5$ & 2.5 & 0.71 & - \\
\hline 15 & 5.03 & 1.0 & 6.28 & 4.0 & 0.20 & $6.3 / 8.2$ & 4.5 & 0.55 & $<10 \%$ \\
\hline \multicolumn{10}{|c|}{ Paddle frequency $f_{p}=0.7 \mathrm{~Hz}$} \\
\hline 15 & 3.70 & 0.7 & 4.4 & 1.97 & 0.073 & $1.2 / 1.4$ & 1.1 & 0.78 & No breaking \\
\hline 20 & 5.03 & 0.7 & 4.4 & 1.97 & 0.10 & $2.2 / 2.6$ & 1.3 & 0.50 & - \\
\hline
\end{tabular}


(a)

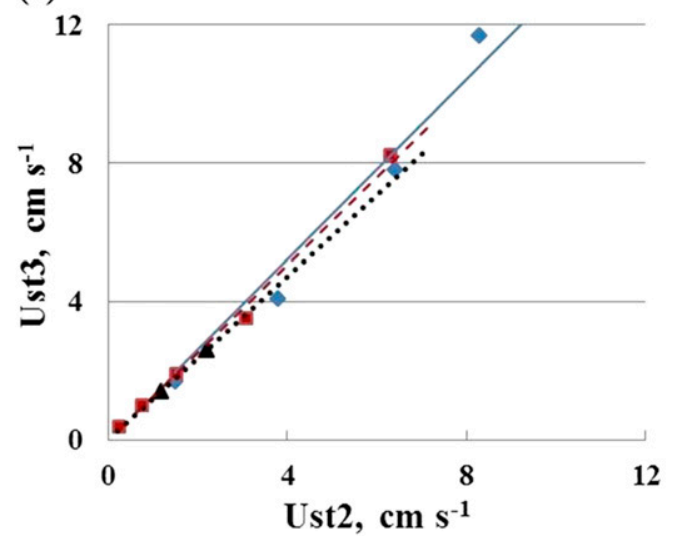

(b)

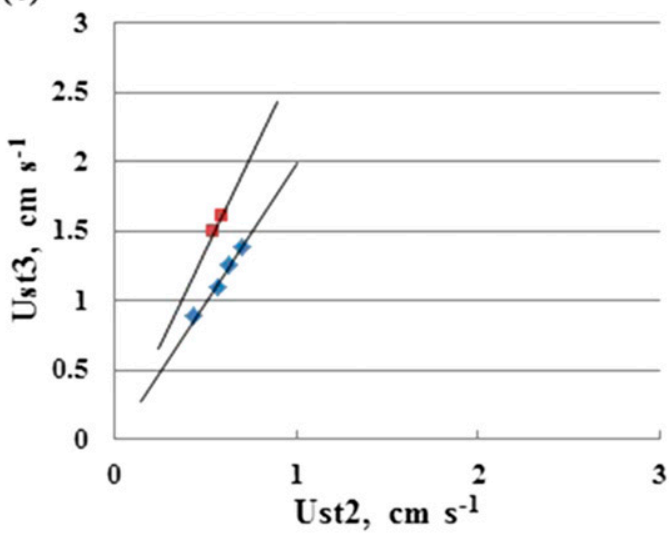

FIG. 3. Relation between the Stokes drift estimations with Eq. (2), Ust2, and with Eq. (3), Ust3. (a) Regular mechanical waves (Table 1$)$ : the solid trend line $\left(y_{1}=1.30 x_{1}\right)$ corresponds to $f_{p}=1.5 \mathrm{~Hz}$; the dashed trend line $\left(y_{2}=\right.$ $\left.1.27 x_{2}\right)$ corresponds to $f_{p}=1.0 \mathrm{~Hz}$; the dotted trend line $\left(y_{3}=1.18 x_{3}\right)$ corresponds to $f_{p}=0.7 \mathrm{~Hz}$. (b) Irregular waves with PM spectra (Table 2): the solid trend line $\left(y_{1}=1.98 x_{1}\right)$ corresponds to $f_{p}=1.5 \mathrm{~Hz}$; the dashed trend line $\left(y_{2}=2.73 x_{2}\right)$ corresponds to $f_{p}=1.0 \mathrm{~Hz}$.

consideration of a wider spectral band than in the previous case (Fig. 2b); therefore, the only explanation for the higher values of $U_{d} / U_{\mathrm{St}}$ is accounting for the small steepness $\sigma$ of irregular waves (see Table 2). Theoretically, it is reasonable to assume that for smaller wave steepness values, the observations of $U_{d}$ should be closer to the theoretical values (Stokes 1847).

\section{c. Combined analysis of mechanical waves}

The presented results are novel, despite numerous empirical reports on the discrepancies between measurements and theoretical values of Stokes drift [e.g., Craik (1982), Breivik et al. (2014), Paprota et al. (2016), and so on, to the review by Bremer and Breivik (2017)]. Notably, for the first time, we obtained empirically quantified ratios $U_{d} / U_{\mathrm{St}}$ for regular and irregular mechanical waves in a tank. These ratios are interesting from three points of view: (i) understanding the reasons for the empirical underestimation of the surface drift currents, (ii) methodical points of measurements, and (iii) identifying possible applications of these results.

Underestimating $U_{d}$ is not new; nevertheless, this issue requires separate consideration. The following reasons for the empirical underestimation of surface drift currents in experiments are well known: (i) the viscosity of the water (which disrupts the potentiality of wave motion), (ii) return currents, (iii) water surface contamination, and (iv) the sailing of floats, including dynamic-pressure braking (McNally and

TABLE 2. Parameters of waves and surface currents for irregular waves; "n-dim" indicates nondimensional.

\begin{tabular}{|c|c|c|c|c|c|c|c|c|c|}
\hline $\begin{array}{l}\text { Paddle } \\
H_{S}(\mathrm{~cm})\end{array}$ & $\begin{array}{c}\text { Wave } \\
\text { amplitude } \\
a(\mathrm{~cm})\end{array}$ & $\begin{array}{c}\text { Peak } \\
\text { frequency } \\
f_{p}(\mathrm{~Hz})\end{array}$ & $\begin{array}{c}\text { Peak } \\
\text { frequency } \\
\omega_{p}\left(\mathrm{~s}^{-1}\right)\end{array}$ & $\begin{array}{c}\text { Peak } \\
\text { wavenumber } \\
k_{p}\left(\mathrm{~m}^{-1}\right)\end{array}$ & $\begin{array}{l}\text { Steep ness } \\
\sigma(\mathrm{n} \text {-dim) }\end{array}$ & $\begin{array}{l}\text { Stokes drift } \\
U_{\mathrm{St}}\left(\mathrm{cm} \mathrm{s}^{-1}\right)\end{array}$ & $\begin{array}{l}\text { Mean drift } \\
U_{d}\left(\mathrm{~cm} \mathrm{~s}^{-1}\right)\end{array}$ & $\begin{array}{r}U_{d} / U_{\mathrm{St}} \\
(\mathrm{n}-\operatorname{dim})\end{array}$ & $\begin{array}{c}\text { Intensity of } \\
\text { breaking (\%) }\end{array}$ \\
\hline \multicolumn{10}{|c|}{ Paddle frequency $f_{p}=1.5 \mathrm{~Hz}, \mathrm{PM}$ spectra } \\
\hline 3 & 0.86 & 1.335 & 8.38 & 7.16 & 0.062 & 0.89 & 0.69 & 0.77 & No breaking \\
\hline 5 & 1.26 & 1.13 & 7.09 & 5.13 & 0.065 & 1.1 & 0.66 & 0.60 & - \\
\hline 7 & 1.56 & 1.01 & 6.34 & 4.1 & 0.064 & 1.26 & 1.11 & 0.88 & - \\
\hline 10 & 1.89 & 0.92 & 5.8 & 3.40 & 0.064 & 1.39 & 1.3 & 0.93 & - \\
\hline 15 & \multicolumn{9}{|c|}{ Data are not reliable due to bad wave formation } \\
\hline \multicolumn{10}{|c|}{$\begin{array}{l}\text { Paddle frequency } f_{p}=1.0 \mathrm{~Hz}, \mathrm{PM} \text { spectra } \\
\text { For } H_{S}<10 \mathrm{~cm} \text { the data are not reliable }\end{array}$} \\
\hline 10 & 1.90 & 0.84 & 5.28 & 2.84 & 0.054 & 1.49 & 1.0 & 0.67 & No breaking \\
\hline 15 & 2.25 & 0.77 & 4.84 & 2.38 & 0.054 & 1.60 & 1.2 & 0.75 & $<10 \%$ \\
\hline \multicolumn{10}{|c|}{$\begin{array}{l}\text { Paddle frequency } f_{p}=1.0 \mathrm{~Hz}, \mathrm{JW} \text { spectra } \\
\text { For } H_{S}<10 \mathrm{~cm} \text { the data are not reliable }\end{array}$} \\
\hline 15 & 3.42 & 0.97 & 6.09 & 3.78 & 0.13 & 4.8 & 2.8 & 0.58 & $100 \%$ \\
\hline \multicolumn{10}{|c|}{$\begin{array}{l}\text { Paddle frequency } f_{p}=0.7 \mathrm{~Hz}, \mathrm{JW} \text { spectra } \\
\text { For } H_{S} \leq 10 \mathrm{~cm} \text { the data are not reliable }\end{array}$} \\
\hline 15 & 2.83 & 0.705 & 4.43 & 2.0 & 0.056 & 2.4 & 1.5 & 0.62 & $<10 \%$ \\
\hline
\end{tabular}


White 1985). The first three reasons were discussed in previous studies (e.g., Longuet-Higgins 1953; Craik 1982; Herterich and Hasselmann 1982; see also Breivik et al. 2014; Paprota et al. 2016). We cannot add more to this discussion, noting that the first two reasons are inevitable and cannot be regulated; however, the third influence can be minimized by cleaning the water surface after each change in frequency, thus the third item should be excluded from the list of reasons discussed. Therefore, we do not dwell on the first three reasons, but the phenomenon of the float sailing is worthy of discussion.

Besides the return current influence (Monismith et al. 2007), which cannot be quantified in our case, in our mind, the sailing of floats on the water surface is the main reason for the abovementioned discrepancy between the experimental and theoretical values of the Stokes drift. To validate this assumption, we tried to regulate the effect of sailing by choosing different sizes of floats. Indeed, when we used plastic balls with a diameter of $5 \mathrm{~mm}$ as the floats, the drift current values were fixed at values in $20 \%-30 \%$ smaller than those for floats that were $3 \mathrm{~mm}$ in diameter. Thereby, the assumption stated above is justified.

The nature of the sailing process is relatively evident. In the case of mechanical waves and spherical floats, the sailing has two negative constituents, namely, the air and the water components. The air component is associated with the air-induced resistance to float propulsion due to the viscosity and negative dynamic pressure of the ambient motionless air. On the other hand, the water sailing is influenced by the same manner due to the rapid decrease in the total drift current with depth, described by profile $U_{d}(z)$. For mechanical waves, this decrease follows from Eq. (3). In the case of wind waves, the rapid decrease in the wind-induced current $U_{d w}(z)$ has been empirically shown by Longo et al. (2012) and described in Polnikov (2011, 2019).

These considerations account for mismatch between theoretical and experimental values, and one may go on to methodical points of the surface-drift current measurements.

From a methodological perspective, a number of previous experiments (e.g., Wu 1975, 1983; Zavadsky and Shemer 2017), in which the volumetric surface floats were used, have suffered from the abovementioned problems, resulting in the underestimation of surface drift in these experiments. Therefore, it is important to make certain methodological changes when obtaining future measurements. To avoid the sailing of floats, it is reasonable to use floats that are fully flat. For example, floats could be pieces of thin flexible tissue (similar to gauze) with a net density less than that of water. Such experiments have been performed in the past (see references in Tsahalis 1979), and they should be planned in the future to further improvement the results presented above.

Another alternative approach involves using PIV methods (e.g., Longo et al. 2012; Paprota et al. 2016) or the laserwarming method (thermal-marking velocimetry) (Savelyev et al. 2012) to measure surface drift currents. However, due to technical reasons related to the optical equipment location, such methods are mainly appropriate for small tanks. But in such cases, the tank boundaries could result in additional sources of errors. Another problem associated with using the optical methods to obtain current measurements in tanks is the restriction to equipment location and movement along a tank.
Ideally, technical progress will help overcome the mentioned methodological problems.

In practical applications, the obtained results can be used as the first guess for expected drift current estimates in the engineering of harbors and other marine infrastructure, taking in mind the influence by swell. On other hand, from the physical point of view, the presented results are useful for accurately estimating the observed wind-induced drift currents $U_{d w}$, based on Eq. (4). It is this idea (rather than simple estimating ratio $U_{d} / U_{\mathrm{St}}$ ) was stimulating our measurements of surface drift currents $U_{d}$ for mechanical waves, the results of which will be actively used below in the data analysis for wind waves.

\section{Wind waves: Results and analysis}

The results for wind waves are summarized in Table 3, where the following notations are used: $\sigma=a k_{p}$ is the mean wave steepness, $A=g /\left(\omega_{p} u_{*}\right)$ is the wave age, and $u_{*}$ is the friction velocity in the air. The last column in Table 3 is the main result of this work, demonstrating the correspondence between empirical and parametrical values of the ratio $U_{d w} / u_{*}$. We return to its analysis later, after considering the accuracy of the results.

\section{a. Analysis of the accuracy of the results}

First, let us estimate the accuracy of the results starting with the wind parameter $u_{*}$. The friction velocity $u_{*}$ is usually obtained from Pitot tube measurements based on the traditional assumption of a logarithmic wind profile (Wu 1975; Longo 2012; Zavadsky and Shemer 2017). In our case, only three upper tubes of the PT set provide relatively accurate estimations of $u_{*}$. The mean error of these estimations, obtained using Microsoft Excel, is 5\%-7\%. It is important to note that the values of $u_{*}$ (as well as $U_{d}$ ) reasonably increase with the fetch (in 1.5-2 times), what is typical for the tank measurements (Toba 1973; Longo 2012; Longo et al. 2012). This fact should be taken into account to obtain accurate values of $U_{d w} / u_{*}$ based the data obtained in different parts of a long tank.

The wave parameters $\omega_{p}=2 \pi f_{p}, \sigma$, and $A$ were estimated with very high accuracy (errors less than $1 \%$ ) from the wave spectra calculated within the MATLAB software (see section 2). For the completeness of the wind-wave spectra description, their spatial evolution is presented in Fig. 4. Due to a wide spectral band for wind waves, using Eq. (3) is quite important for estimation of theoretical value for the Stokes drift. In our case, these values have errors of approximately $5 \%-7 \%$, what is defined mainly by the value of the upper limit of the integral in Eq. (3). It was chosen to be $15 \mathrm{~Hz}$ due to the technical restrictions of the WGs used. As shown in Fig. 4, the wave spectra are radically changing along the tank (this feature was ignored for mechanical waves), therefore the theoretical estimates of $U_{\mathrm{St}}$ are also varying, which plays a crucial role in the correct estimating the wind-induced drift currents $U_{d w}$ based on using Eq. (3).

The visually obtained float movement timing resulted in the error on the order of $10 \%$ for the measured total mean drift $U_{d}$. In turn, the intensity of wave breaking was estimated (by two persons in parallel) with the same error. These values influence 
TABLE 3. Parameters of wind, waves and surface currents for wind waves. Note that the $\leftrightarrow$ sign separates the empirical and parametrical values $U_{\mathrm{dw}} / u_{*}$ estimated with Eq. (8); "n-dim” indicates nondimensinonal.

\begin{tabular}{|c|c|c|c|c|c|c|c|c|c|}
\hline $\begin{array}{c}\text { Fan wind } \\
\qquad \begin{array}{c}W \\
\left(\mathrm{~m} \mathrm{~s}^{-1}\right)\end{array}\end{array}$ & $\begin{array}{c}\text { Friction } \\
\text { velocity } \\
u_{*}\left(\mathrm{~cm} \mathrm{~s}^{-1}\right)\end{array}$ & $\begin{array}{c}\text { Peak } \\
\text { frequency } \\
\omega_{p}\left(\mathrm{~s}^{-1}\right)\end{array}$ & $\begin{array}{c}\text { Steepness } \\
\sigma(\text { n-dim })\end{array}$ & $\begin{array}{l}\text { Wave age } \\
A \text { (n-dim) }\end{array}$ & $\begin{array}{c}\text { Stokes drift } \\
U_{\mathrm{St}} \\
\left(\mathrm{cm} \mathrm{s}^{-1}\right)\end{array}$ & $\begin{array}{c}\text { Mean drift } \\
U_{d} \\
\left(\mathrm{~cm} \mathrm{~s}^{-1}\right)\end{array}$ & $\begin{array}{l}\text { Wind drift } \\
U_{\mathrm{dw}} \\
\left(\mathrm{cm} \mathrm{s}^{-1}\right)\end{array}$ & $\begin{array}{c}\text { Intensity of } \\
\text { breaking, } \\
(\mathrm{Br} ; \%)\end{array}$ & $\begin{array}{c}\text { Empirical } \leftrightarrow \\
\text { parametric } \\
U_{d w} / u_{*}\end{array}$ \\
\hline & \multicolumn{9}{|c|}{ Measurements at $\mathrm{P} 1=9 \mathrm{~m}$} \\
\hline 4 & 8.2 & 28.1 & 0.27 & 4.3 & 2.38 & 12.5 & 10.1 & No breaking & $1.2 \leftrightarrow 1.16$ \\
\hline 6 & 11.7 & 24.9 & 0.29 & 3.4 & 4.92 & 16.7 & 11.8 & - & $1.0 \leftrightarrow 1.1$ \\
\hline 8 & 14.4 & 20.1 & 0.34 & 3.4 & 6.89 & 23.5 & 16.6 & - & $1.1 \leftrightarrow 1.1$ \\
\hline 10 & 20.4 & 18.0 & 0.33 & 2.72 & 9.58 & 30.8 & 21.2 & $5 \%$ & $1.1 \leftrightarrow 0.94$ \\
\hline \multirow[t]{2}{*}{12} & 28.2 & 17.1 & 0.37 & 2.0 & 12.4 & 36.4 & 24.0 & $10 \%$ & $0.86 \leftrightarrow 0.84$ \\
\hline & \multicolumn{9}{|c|}{ Measurements at $\mathrm{P} 2=12 \mathrm{~m}$} \\
\hline 4 & 9.5 & 22.0 & 0.26 & 4.7 & 4.54 & 13.5 & 8.95 & No breaking & $0.98 \leftrightarrow 1.2$ \\
\hline 6 & 14.2 & 18.1 & 0.26 & 3.8 & 5.93 & 17.6 & 11.7 & - & $0.82 \leftrightarrow 0.99$ \\
\hline 8 & 17.7 & 16.6 & 0.28 & 3.34 & 7.77 & 22.2 & 14.4 & - & $0.81 \leftrightarrow 0.93$ \\
\hline 10 & 25.4 & 14.6 & 0.29 & 2.64 & 10.6 & 33.3 & 22.7 & $10 \%$ & $0.89 \leftrightarrow 0.87$ \\
\hline \multirow[t]{2}{*}{12} & 34.3 & 12.9 & 0.28 & 2.2 & 13.9 & 40 & 26.1 & $20 \%$ & $0.78 \leftrightarrow 0.82$ \\
\hline & \multicolumn{9}{|c|}{ Measurements at $\mathrm{P} 4=22 \mathrm{~m}$} \\
\hline 4 & 12.5 & 16.2 & 0.19 & 4.8 & 3.35 & 14.3 & 11.0 & No breaking & $0.88 \leftrightarrow 0.91$ \\
\hline 6 & 17.5 & 14.1 & 0.21 & 3.98 & 5.25 & 20.0 & 14.7 & - & $0.84 \leftrightarrow 0.83$ \\
\hline 8 & 27.8 & 12.9 & 0.25 & 2.73 & 7.9 & 26.7 & 18.9 & - & $0.68 \leftrightarrow 0.68$ \\
\hline 10 & 40.0 & 11.5 & 0.27 & 2.13 & 11.5 & 40 & 28.5 & $15 \%$ & $0.71 \leftrightarrow 0.71$ \\
\hline 12 & 53.0 & 10.8 & 0.29 & 1.7 & 15.5 & 50 & 34.5 & $20 \%$ & $0.65 \leftrightarrow 0.7$ \\
\hline
\end{tabular}

the accuracy of the empirical results for the wind-induced drift currents $U_{d w}$.

\section{b. Analysis of the main results}

In the case of wind waves, we estimated the wind-induced drift currents as the difference between the measured value $U_{d}$ and the theoretically estimated value $U_{\mathrm{St}}$ by assuming that the positive floats sailing in the air (due to the wind) is compensated for by the negative floats sailing in the water. This assumption was verified by the measurements with plastic balls of different diameters ( 3 and $5 \mathrm{~mm}$ ) used as the floats. This means that the values of $U_{d w}$ presented in Table 3 are the smallest possible values, taking in mind the results for mechanical waves discussed above in section 3 a.

Based on Table 3 , the determined empirical ratio $U_{d w} / u_{*}$ varies in the wide range of $0.65-1.2$, which corresponds to the range presented in the literature (see section $1 \mathrm{~b}$ ). This peculiarity of our results will be separately discussed below in section 5, but here, the main attention is paid on identifying the proper parameterization for the dependence of ratio $U_{d w} / u_{*}$ on the parameters of the system under consideration. The theoretical basis for such parameterization is the following.

According to the theoretical model of the wind-induced drift currents proposed in a previous study (Polnikov 2019), one may expect $U_{d w} / u_{*}$ to be dependent on the following wave parameters: steepness $\sigma=a k_{p}$, wave age $A=g /\left(\omega_{p} u_{*}\right)$, and breaking intensity (Br). After several attempts (see the assumption for the general approximation below), we obtained the following relation:

$$
U_{d w} / u_{*}=\mathrm{Br}+\sigma A
$$

In a graphic form, the relation between the empirical and parametrical values of $U_{d w} / u_{*}$ is shown in Fig. 5. From Table 3 and Fig. 5, it is seen that parameterization (8) is valid with a mean error of approximately $10 \%$, which is very good for experimental data.

Equation (8) provides the dependence relation between the wind-induced surface drift currents and the wind and wave parameters, which is the main aim of this paper. This dependence is

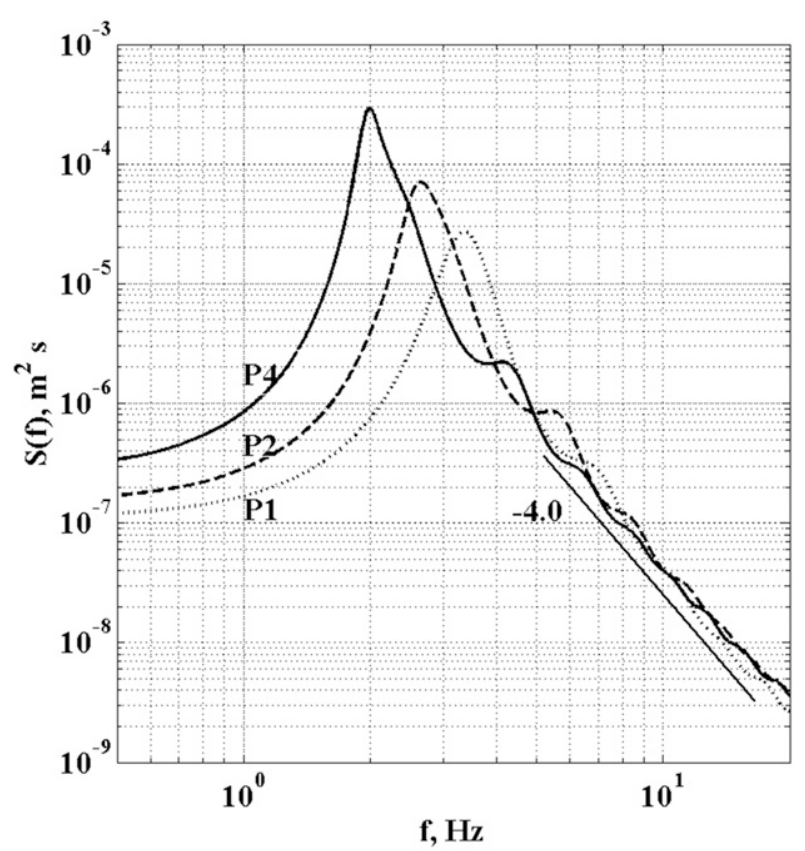

FIG. 4. Wind-wave spectra at points $\mathrm{P} 1, \mathrm{P} 2$, and $\mathrm{P} 4$ for the run with $W=8 \mathrm{~m} \mathrm{~s}^{-1}$. The straight line with digits shows the frequencydecay law of spectra in the tail range (the error is $\sim 3 \%$ ). 


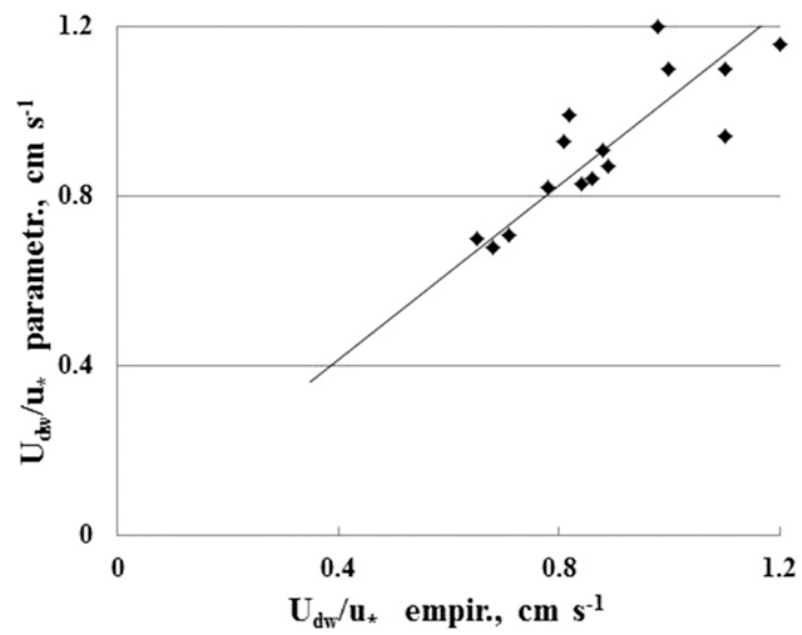

FIG. 5. Correspondence between empirical and parametrical values of ratio $U_{d w} / u_{*}$ estimated with Eq. (8) for wind waves. The trend line is described by the equation $y=1.03 x$ with a mean accuracy of $10 \%$.

established for the first time during a long history of empirical investigations (section 1a). Below, it will be shown that ratio (8) corresponds relatively well to the results published earlier for the tank measurements (Wu 1975; Longo 2012; Longo et al. 2012; Zavadsky and Shemer 2017). In the future, parameterization (8) could be checked for the field data based on accurate measurements of wind and wave parameters.

\section{Discussion}

In this section, we discuss the following points: (i) the impact of tank dimensions and wind variations along a tank on the accuracy of estimates of $u_{*}$; (ii) the role of theoretical estimates of the Stokes drift in assessing empirical measurements; (iii) the application of a parametric representation of ratio $U_{d w} / u_{*}$ for other empirical data; and (iv) adjusting the methodology to minimize the float sailing in traditional and alternative approaches.

First, we should mention that the small dimensions of tanks used in some studies (Wu 1975; Longo et al. 2012; Zavadsky and Shemer 2017) could reasonably restrict the accuracy of the wind and wave measurements. Evidently, in such cases, the upper and lateral boundaries of tanks are the sources of errors in the wind profile measurements and, consequently, the estimations of $u_{*}$. The main reason for these errors is that the theoretical logarithmic profile cannot be fully formed in small tanks (Troitskaya et al. 2012), which leads to overestimating the wind gradient $\partial W / \partial z$, and consequently, overestimating the friction velocity $u_{*}$. For wind waves with mean heights $a>$ 2-3 cm (corresponding to significant heights $H_{S}>6-9 \mathrm{~cm}$ ) in tanks with air-part heights less than $0.5 \mathrm{~m}$ (e.g., Toba 1973; Wu 1975; Longo 2012; Zavadsky and Shemer 2017), due to the reasons mentioned, the values of $u_{*}$ could be overestimated. Moreover, tanks with working lengths less than 5-8 m, they usually use only one point for the wind profile measurements, thereby neglecting information about the wind profile changes along a tank. Additionally, it is difficult to provide a wide range of wave parameters in a small tank, which restricts the possibility of revealing the dependence of drift currents on the parameters of the wave state. All these factors explain why we have observed greater values of $U_{d w} / u_{*}$ than those previously reported, and established the dependence of $U_{d w}$ on the windwave parameters, which was not fixed in the earlier laboratory work (Wu 1975; Longo 2012; Longo et al. 2012; Zavadsky and Shemer 2017).

Regarding the Stokes drift estimations, in the presence of mechanical waves, the current measurements inevitably underestimate the wave-induced drift at the water surface with respect to the theoretical value $U_{\text {St }}$. In turn, as shown in section 3, the theoretical estimates of $U_{\mathrm{St}}$ with Eq. (2) that are typically used in practice (e.g., Toba 1973; Wu 1975; Longo 2012; Longo et al. 2012) are incorrect. Therefore, the reported ratio between $U_{\mathrm{St}}$ and $U_{d w}$ was underestimated, the value of which was fixed at approximately 15\% (Toba 1973; Wu 1975; Longo et al. 2012). The more accurate estimates, especially in the presence of wind waves with wideband spectra, can be obtained with using Eq. (3). In such a case, as shown in Table 3 , the ratio $U_{\mathrm{St}} / U_{d w}$ could reach values of $35 \%-40 \%$. This circumstance is very important from a practical perspective. Of course, all these results should be checked again in future experiments conducted with highly accurate measurements.

Regarding the wind-induced drift parameterization, the general assumption is of the form

$$
U_{d w}=\left[C_{b} \mathrm{Br}^{k}+C_{w} \sigma^{m}(A)^{n}\right] u_{*},
$$

where factors $C_{b}, C_{w}$, and power parameters $k, m$, and $n$ are the fitting dimensionless constants. Based on the trial-and-error method, we found that the simplest version of Eq. (9) has the form of Eq. (8), when all the parameters are equal to unity. According to Table 3, Eq. (8) provides a relatively good approximation, with a mean error for the value $U_{d w} / u_{*}$ of $10 \%$. The only weakness of parameterizations (8) and (9) is their dependence on the breaking intensity, which is difficult to estimate empirically. This issue requires additional consideration in the future.

At meanwhile, it is interesting to apply parameterization (8) to the other known results. For example, for results of the classical work by Wu (1975), we obtained the following findings. From Figs. 2 and 3 in Wu (1975), typical values for the wave parameters are $a=0.02-0.03 \mathrm{~m}, \omega_{p}=12.5 \mathrm{~s}^{-1}$, while $u_{*}=0.5-0.6 \mathrm{~m} \mathrm{~s}^{-1}$, in the case of the tunnel wind $W=10 \mathrm{~m} \mathrm{~s}^{-1}$. These values yield $\sigma A=a \omega_{p} / u_{*} \approx 0.5$, which, according to Eq. (8) in the case of no breaking, corresponds very well to the value $U_{d w} \approx 0.53 u_{*}$ reported in $\mathrm{Wu}$ (1975).

Addressing the data from Longo (2012) and Longo et al. (2012), we obtained the following findings. From Tables 1 and 3 of Longo (2012), the typical wave parameters are $a \approx 0.002-$ $0.003 \mathrm{~m}$ and $\omega_{p}=30-40 \mathrm{~s}^{-1}$, which resulting in $\sigma \approx 0.2$, for a tunnel wind of $W=10.9 \mathrm{~m} \mathrm{~s}^{-1}$. From Table 1 of Longo et al. (2012), in this case, $u_{*}=0.6-0.7 \mathrm{~m} \mathrm{~s}^{-1}$. These values yield $\sigma A \approx$ 0.2. According to Longo et al. (2012), the breaking intensity $\mathrm{Br}$ is approximately of $20 \%$ (which is very likely for $\sigma \approx 0.2$ ), thus 
parameterization (8) results in the value $U_{d w} \approx 0.4 u_{*}$ reported in Longo et al. (2012).

In Zavadsky and Shemer (2017), the analogous data for wind $W=10.5 \mathrm{~m} \mathrm{~s}^{-1}$ are as follows: $a \approx 0.01 \mathrm{~m}, \omega_{p} \approx 15 \mathrm{~s}^{-1}$, and $u_{*} \approx 0.7 \mathrm{~m} \mathrm{~s}^{-1}$ (from their Fig. 8). According to (8), these values lead to the estimate $U_{d w} \approx 0.3 u_{*}$, which is close to the reported value in this paper.

As one can see, parameterization (8) works well in all these cases. Similar examples can be explored for the other experimental works mentioned above if the proper data are supplied.

Finally, the methodology should be discussed. Naturally, using hard surface floats to measure surface drift currents seems obsolete. The more advanced methods are PIV (e.g., Longo 2012; Longo et al. 2012; Paprota et al. 2016) or laserwarming methods (Savelyev et al. 2012), although they cannot be used in large tanks due to the need to apply many optical devices through transparent tank boundaries and restrictions related to moving optical equipment throughout the tank. Most laboratories have no such equipment at their disposal. Nevertheless, traditional floats can still provide reasonable results.

The main problem, in this case, is the sailing of floats, as discussed in detail in section 3c. This problem could be overcome if one uses appropriate floats. The solution is to use flat floats in the form of pieces of thin flexible tissue (like gauze) with a net density less than that of water. If the width of the tissue is $0.1 \mathrm{~mm}$, the sailing of the floats will be negligibly small; thus, they would not be disturbed by the wind or the vertical shear of drift currents. In such a case, the results of the drift measurements would be more accurate than in most other cases, what suggests that one could plan similar experiments in the near future. Thus, the best choice is to use tanks with lengths greater than $30 \mathrm{~m}$, widths greater than $1 \mathrm{~m}$ and air part of approximately $1 \mathrm{~m}$ or more to ensure correct estimations of the friction velocity $u_{*}$, as discussed above. Under these conditions, the results presented in this paper could be further evaluated.

\section{Conclusions}

Finally, we state the following conclusions.

First, the surface drift currents $U_{d}$ induced by mechanical waves in a tank were measured with plastic surface floats and compared with the Stokes drift at the surface $U_{\mathrm{St}}$ estimated by Eq. (3). The ratio $U_{d} / U_{\mathrm{St}}$ varies in the range of $0.5-0.93$ and slightly increases with decreasing wave steepness, with no visible dependence on the breaking intensity. The reduction in empirical values of $U_{d}$ with respect to the theoretical value $U_{\mathrm{St}}$ is mainly due to the following reasons: return currents, fluid viscosity, and the sailing of floats in the air and in the water. The significant effect of the latter reason could be minimized by using flat and flexible tissue floats or by employing modern optical methods to collect surface current measurements.

Second, in the case of wind waves, the wind-induced part of the surface drift $U_{d w}$ was estimated by subtracting the theoretical value for the Stokes drift $U_{\mathrm{St}}$ found with Eq. (3) from the measured total drift $U_{d}$ at three locations along the tank for a series of wind speeds. These values of $U_{d w}$ were compared with the friction velocity $u_{*}$ measured at the same three locations.
The obtained ratio $U_{d w} / u_{*}$ varies systematically in the range of $0.65-1.2$.

Third, the obtained ratio $U_{d w} / u_{*}$ was parameterized by Eq. (8). For the first time, the dependence of the wind-induced drift current $U_{d w}$ on the wave state parameters was found. The parameterization (8) corresponds to observations with a mean error of less than $10 \%$. The application of parameterization (8) to other known results for measurements of $U_{d w}$ and $u_{*}$ in tanks showed encouraging results (see section 5).

Acknowledgments. The authors are grateful to $\mathrm{PhD}$ student Shumin Jiang and graduate students Xue Wang and Chao Li for their help with measurements and data processing. The work was supported by the National Key Research and Development Program of China under Grant 2017YFC1404005, the Russian Foundation for Basic Research Grant 19-04-00161, and the Natural Science Foundation of China under Grants 41821004 and U160640.

Data availability statement. The raw measurement data are available from the corresponding author (V. Polnikov).

\section{REFERENCES}

Anis, A., and J. N. Moum, 1995: Surface wave-turbulence interactions: Scaling $\varepsilon(z)$ near the sea surface. J. Phys. Oceanogr., 25, 2025-2045, https://doi.org/10.1175/1520-0485(1995)025<2025: SWISNT $>2.0$.CO;2.

Babanin, A. V., 1988: Relationship between the parameters of the surface drift current and the determining wind in the northwestern part of the Black Sea (in Russian). Mar. Hydrophys. J., 4, 55-58.

— induced by non-breaking surface waves. J. Phys. Oceanogr., 39, 2675-2679, https://doi.org/10.1175/2009JPO4202.1.

_ , and D. Chalikov, 2012: Numerical investigation of turbulence generation in non-breaking potential waves. J. Geophys. Res., 117, C00J17, https://doi.org/10.1029/2012JC007929.

Benilov, A. Y., 2012: On the turbulence generated by the potential surface waves. J. Geophys. Res., 117, C00J30, https://doi.org/ 10.1029/2012JC007948.

Breivik, O., J. Bidlot, and P. A. E. M. Janssen, 2014: Approximate Stokes drift profiles in deep water. J. Phys. Oceanogr., 44, 2433-2445, https://doi.org/10.1175/JPO-D-14-0020.1.

Bremer, T. S., and Ø. Breivik, 2017: Stokes drift. Philos. Trans. Roy. Soc., 376A, 20170104, http://doi.org/10.1098/rsta.2017.0104.

Bühler, O., 2014: Waves and Mean Flows. 2nd ed. Cambridge University Press, 360 pp.

Bye, J., 1988: The coupling of wave drift and wind velocity profiles. J. Mar. Res., 46, 457-472, https://doi.org/10.1357/ 002224088785113559.

Chalikov, D., and S. Rainchik, 2011: Coupled numerical modelling of wind and waves and theory of the wave boundary layer. Bound.-Layer Meteor., 138, 1-41, https://doi.org/10.1007/ s10546-010-9543-7.

Churchill, J. H., and G. T. Csanady, 1983: Near-surface measurements of quasi-Lagrangian velocities in open water. J. Phys. Oceanogr., 13, 1669-1680, https://doi.org/10.1175/1520-0485(1983)013<1669: NSMOQL $>2.0 . C O ; 2$.

Clarke, A. J., and S. V. Gorder, 2018: The relationship of nearsurface flow, Stokes drift and the wind stress. J. Geophys. Res. Oceans, 123, 4680-4692, https://doi.org/10.1029/2018JC014102. 
Constantin, A., 2006: The trajectories of particles in Stokes waves. Invent. Math., 166, 523-535, https://doi.org/10.1007/s00222006-0002-5.

Craik, A. D. D., 1982: The drift velocity of water waves. J. Fluid Mech., 116, 187-205, https://doi.org/10.1017/S0022112082000421.

Herterich, K., and K. Hasselmann, 1982: The horizontal diffusion of tracers by surface waves. J. Phys. Oceanogr., 12, 704-711, https://doi.org/10.1175/1520-0485(1982)012<0704: THDOTB $>2.0 . \mathrm{CO} ; 2$.

Huang, N. E., 1979: On surface drift currents in the ocean. J. Fluid Mech., 91, 191-208, https://doi.org/10.1017/S0022112079000112.

Janssen, P. A. E. M., 2004: The Interaction of Ocean Waves and Wind. Cambridge University Press, 308 pp.

Kitaigorodskii, S., M. A. Donelan, J. L. Lumley, and E. A. Terray, 1983: Wave-turbulence interactions in the upper ocean. Part II: Statistical characteristics of wave and turbulent components of the random velocity field in the marine surface layer. J. Phys. Oceanogr., 13, 1988-1999, https://doi.org/10.1175/ 1520-0485(1983)013<1988:WTIITU>2.0.CO;2.

Komen, G. I., L. Cavaleri, M. Donelan, K. Hasselmann, S. Hasselmann, and P. A. E. M. Janssen, 1994: Dynamics and Modelling of Ocean Waves. Cambridge University Press, $554 \mathrm{pp}$.

Kudryavtsev, V. N., V. Shrira, V. Dulov, and V. V. Malinovsky, 2008: On the vertical structure of wind-driven sea currents. J. Phys. Oceanogr., 38, 2121-2144, https://doi.org/10.1175/2008JPO3883.1.

Longo, S., 2012: Wind-generated water waves in a wind tunnel: Free surface statistics, wind friction and mean air flow properties. Coastal Eng., 61, 27-41, https://doi.org/10.1016/ j.coastaleng.2011.11.008.

— L. Chiapponi, M. Clavero, T. Mäkel, and D. Liang, 2012: Study of the turbulence in the air-side and the water-side boundary layers in experimental laboratory wind induced surface waves. Coastal Eng., 69, 67-81, https://doi.org/10.1016/j.coastaleng.2012.05.012.

Longuet-Higgins, M., 1953: Mass transport in water waves. Philos. Trans. Roy. Soc. London, 245A, 535-581, https://doi.org/ 10.1098/rsta.1953.0006.

Lumley, J. L., and E. A. Terray, 1983: Kinematics of turbulence convected by a random wave field. J. Phys. Oceanogr., 13, 2000-2007, https://doi.org/10.1175/1520-0485(1983)013<2000: KOTCBA $>2.0 . \mathrm{CO} ; 2$.

Malinovsky, V. V., V. A. Dulov, A. E. Korinenko, A. N. Bol'shakov, and V. E. Smolov, 2007: Field investigations of the drift of artificial thin films on the sea surface. Izv. Atmos. Oceanic Phys., 43, 103-111, https://doi.org/10.1134/S0001433807010124.

McNally, G. J., and W. B. White, 1985: Wind driven flow in the mixed layer observed by drifting buoys during autumnwinter in the midlatitude North Pacific. J. Phys. Oceanogr., 15, 684-694, https://doi.org/10.1175/1520-0485(1985)015<0684: WDFITM $>2.0 . \mathrm{CO} ; 2$.

Mellor, G. L., 2001: One-dimensional, ocean surface layer modelling: A problem and a solution. J. Phys. Oceanogr., 31, 790-809, https://doi.org/10.1175/1520-0485(2001)031<0790: ODOSLM $>2.0 . \mathrm{CO} ; 2$.

Monismith, S. G., E. A. Cowen, H. M. Nepf, J. Magnaudet, and L. Thais, 2007: Laboratory observations of mean flows under surface gravity waves. J. Fluid Mech., 573, 131-147, https:// doi.org/10.1017/S0022112006003594.

Paprota, M., W. Sulisz, and A. Reda, 2016: Experimental study of wave-induced mass transport. J. Hydraul. Res., 54, 423-434, https://doi.org/10.1080/00221686.2016.1168490.

Phillips, O. M., 1977: The Dynamics of the Upper Ocean. 2nd ed. Cambridge University Press, 336 pp.

Polnikov, V. G., 2011: Integrated model for a wave boundary layer. Mar. Sci., 1, 10-21, https://doi.org/10.5923/j.ms.20110101.02.

—_, 2019: A semi-phenomenological model for wind-induced drift currents. Bound.-Layer Meteor., 172, 417-433, https:// doi.org/10.1007/s10546-019-00456-1.

Rascle, N., and F. Ardhuin, 2009: Drift and mixing under the ocean surface revisited: Stratified conditions and model-data comparisons. J. Geophys. Res., 114, C02016, https://doi.org/ 10.1029/2007JC004466.

Savelyev, I. B., E. Maxeiner, and D. Chalikov, 2012: Turbulence production by nonbreaking waves: Laboratory and numerical simulations. J. Geophys. Res., 117, C00J13, https://doi.org/ 10.1029/2012JC007928.

Shemdin, O. H., 1972: Wind-generated current and phase speed of wind waves. J. Phys. Oceanogr., 2, 411-419, https://doi.org/ 10.1175/1520-0485(1972)002<0411:WGCAPS > 2.0.CO;2.

Stokes, G. G., 1847: On the theory of oscillatory waves. Trans. Cambridge Philos. Soc., 8, 441-455.

Sullivan, P. P., and J. C. McWilliams, 2010: Dynamics of winds and currents coupled to surface waves. Annu. Rev. Fluid Mech., 42, 19-42, https://doi.org/10.1146/annurev-fluid121108-145541.

Teixeira, M. A. C., 2018: A model for the wind-driven current in the wavy oceanic surface layer: Apparent friction velocity reduction and roughness length enhancement. J. Phys. Oceanogr., 48, 2721-2736, https://doi.org/10.1175/JPO-D-18-0086.1.

_ and S. E. Belcher, 2002: On the distortion of turbulence by a progressive surface wave. J. Fluid Mech., 458, 229-267, https:// doi.org/10.1017/S0022112002007838.

Toba, Y., 1973: Local balance in the air-sea boundary processes. III: On the spectrum of wind waves. J. Oceanogr. Soc. Japan, 29, 209-220, https://doi.org/10.1007/BF02108528.

Troitskaya, Y. I., D. A. Sergeev, A. A. Kandaurov, G. A. Baidakov, M. A. Vdovin, and V. I. Kazakov, 2012: Laboratory and theoretical modeling of air-sea momentum transfer under severe wind conditions. J. Geophys. Res., 117, C00J21, https://doi.org/ 10.1029/2011JC007778.

Tsahalis, D. T., 1979: Theoretical and experimental study of wind- and wave-induced drift. J. Phys. Oceanogr., 9, 1243-1257, https:// doi.org/10.1175/1520-0485(1979)009<1243:TAESOW>2.0.CO;2.

Wu, J., 1975: Wind-induced drift currents. J. Fluid Mech., 68, 49-70, https://doi.org/10.1017/S0022112075000687.

_ 1983: Sea-surface drift currents induced by wind and waves. J. Phys. Oceanogr., 13, 1441-1451, https://doi.org/10.1175/ 1520-0485(1983)013<1441:SSDCIB > 2.0.CO;2.

Zavadsky, A., and L. Shemer, 2017: Water waves excited by nearimpulsive wind forcing. J. Fluid Mech., 828, 459-495, https:// doi.org/10.1017/jfm.2017.521. 